\title{
Clinical Experience with Gastrocnemius Recession Surgery in Chronic Plantar Fasciitis
}

\author{
Rahul Upadhyay ${ }^{1}$, Jitesh Jain ${ }^{2}$, Divyanshu Goyal ${ }^{3}$
}

\begin{abstract}
Aim and objective: In this study, we evaluated functional outcomes in patients treated with gastrocnemius recession for chronic plantar fasciitis due to isolated gastrocnemius tightness.

Materials and methods: In this prospective study, 20 patients with chronic plantar fasciitis due to isolated gastrocnemius tightness were included. Non-conservative treatment was given to all patients for 9 months. After the trial of conservative treatment, 20 patients who did not respond well were operated for gastrocnemius (18 open and 2 endoscopic procedures). American Orthopaedic Foot and Ankle Score (AOFAS) was calculated at 6 weeks, 3 months, 6 months, and 9 months. Visual analog score (VAS) was recorded at 9 months. At final follow-up at 9 months, patients were asked to choose satisfaction level in terms of high/partial/low and would they recommend this surgery to their friends and if they would go for the same for the opposite limb.

Results: Visual analog score improved from 7.2 to $1.2(p$ value $<0.0001$ ). The difference of the AOFAS at final follow-up (9 months) was found to be highly significant with a pre-op score of 49.4 to a final score as high as 93.3 ( $p$ value $<0.0001$ ). Patient satisfaction level was calculated with relation to the AOFAS. High satisfaction was observed in 18 patients and partial satisfaction in 2 patients.

Conclusion: Gastrocnemius recession done for isolated gastrocnemius tightness causing chronic plantar fasciitis provides statistically significant improvement without any major complications with a short recovery period.

Keywords: American Orthopaedic Foot and Ankle Score Ankle-hindfoot score, Functional, Gastrocnemius recession, Gastrocnemius tightness, Heel pain, Plantar fasciitis, Visual analog pain score, Visual analog score.

Journal of Foot and Ankle Surgery (Asia Pacific) (2022): 10.5005/jp-journals-10040-1177
\end{abstract}

\section{INTRODUCTION}

Heel pain is one of the most common outpatient presentations in the orthopedics department. Treating heel pain can be challenging at times due to its wide variety of causes including plantar fasciitis, Achilles tendinopathy, retrocalcaneal bursitis, and nerve entrapment. Plantar fasciitis is a common cause of heel pain affecting all age groups. Around 1 million people visit orthopedic clinics annually in the United States for plantar fasciitis ${ }^{1}$ and the number would be much more in a populated country like India.

Plantar fasciitis is diagnosed with proper history taking and clinical examination. Routine radiographs are required to rule out other causes like Haglund's disease. Most of the patients complain of heel pain in the morning when they step out of the bed or when they walk after sitting for a long time. Riddle et al. ${ }^{1}$ found an independent association of plantar fasciitis with obesity (BMI >30), with people who have work-related weight-bearing for long hours and with reduced ankle dorsiflexion (gastrocnemius or gastrosoleus tightness).

The pathogenesis of plantar fasciitis most widely accepted is repetitive activation of windlass mechanism and tensile overload leads to micro-tears and activates an inflammatory response. This in turn causes chronic degenerative changes in fascia leading to the symptoms.

Different modalities of treatment are available including both nonoperative and operative. Nonoperative treatment is the treatment of choice and around $90 \%$ of the patients are relieved by it if continued for around 9 months. It ranges from stretching exercises for gastrocnemius and plantar fascia, night splints, orthotics, non-steroidal anti-inflammatory drugs (NSAIDs), corticosteroid injections, and plasma-rich protein (PRP) injections to botulinum

\footnotetext{
${ }^{1}$ Department of Orthopaedics, Foot and Ankle Injury Centre, Jaipur, Rajasthan, India

${ }^{2}$ Department of Orthopaedics, Apex Hospital, Jaipur, Rajasthan, India ${ }^{3}$ Department of Orthopaedics, SMS Hospital, Jaipur, Rajasthan, India

Corresponding Author: Rahul Upadhyay, Department of Orthopaedics, Foot and Ankle Injury Centre, Jaipur, Rajasthan, India, Phone: +91 9990283055, e-mail: drrahul.smc@gmail.com

How to cite this article: Upadhyay R, Jain J, Goyal D. Clinical Experience with Gastrocnemius Recession Surgery in Chronic Plantar Fasciitis. J Foot Ankle Surg (Asia Pacific) 2022;9(1):21-24.

Source of support: Nil

Conflict of interest: None
}

toxin injections. Those patients who fail to respond to nonoperative treatment even after 9 months are the candidates for operative treatment which includes plantar fasciectomy and gastrocnemius recession. Isolated gastrocnemius contracture has been identified as a major cause of plantar fasciitis. ${ }^{2}$ Gastrocnemius recession has been proposed as a treatment of choice for it. ${ }^{3,4}$

In this study, we evaluate functional outcomes in patients treated with gastrocnemius recession for chronic plantar fasciitis due to isolated gastrocnemius tightness.

\section{Materials and Methods}

After taking ethical committee clearance, the study was conducted from January 2017 to October 2018. We included patients with chronic plantar fasciitis having isolated gastrocnemius tightness visiting our clinic for the first time. We excluded patients who had 
plantar fasciitis due to any other cause, combined gastrosoleus tightness, who had any kind of prior surgery on the foot.

After taking proper history, all these patients were examined for isolated gastrocnemius tightness using the Silfverskiold test. All patients were started with nonoperative treatment consisting of NSAIDs, gastrocnemius stretching exercises, plantar fascia-specific stretching exercises, night splints, orthotics as required by the individual patients. This was continued for 9 months.

Twelve patients were lost to follow-up at the end of 9 months. Visual analog score (VAS), American Orthopaedic Foot and Ankle Score (AOFAS), and patient satisfaction (patients were given the option to choose between high/partial/low) were recorded for the remaining 20 patients. After this evaluation, all patients were given the option for operative treatment i.e., gastrocnemius recession. All 20 patients gave consent for the surgery.

\section{Surgical Technique}

All cases were operated by a single senior surgeon. In all 20 cases, open gastrocnemius recession was done at the level of musculotendinous junction.

After a proper preanesthetic check-up, patients were taken to the operation theater. Spinal anesthesia was given in all cases. Under all aseptic precautions, scrubbing, painting, and draping were done in the lateral position with the affected side up. Surface marking was done for the Achilles tendon and sural nerve (Fig. 1). A 3-4 cm long longitudinal incision was given at a distance of 15-17 cm proximally from the insertion of the Achilles tendon (Fig. 2). This incision was kept a little lateral from the midline. Fascia and paratenon were incised and split longitudinally. The sural nerve was identified and secured. Musculotendinous junction of gastrocnemius was identified and gastrocnemius recession was done until full dorsiflexion at ankle joint was achieved (Fig. 3). Paratenon and fascia were left open while the skin was closed using ethilon 2-0 suture.

Postoperatively, patients were allowed partial weight-bearing in a below-knee cast for 2 weeks. Sutures were removed at 2 weeks. Walking boots were given at 2 weeks and partial weight-bearing and full ankle range of motion (ROM) exercises were advised. At 4 weeks, plantar fascia-specific exercises were started and, at 8 weeks, progressive increase in weight-bearing was allowed. Full ranges of activities were allowed from 12 weeks.

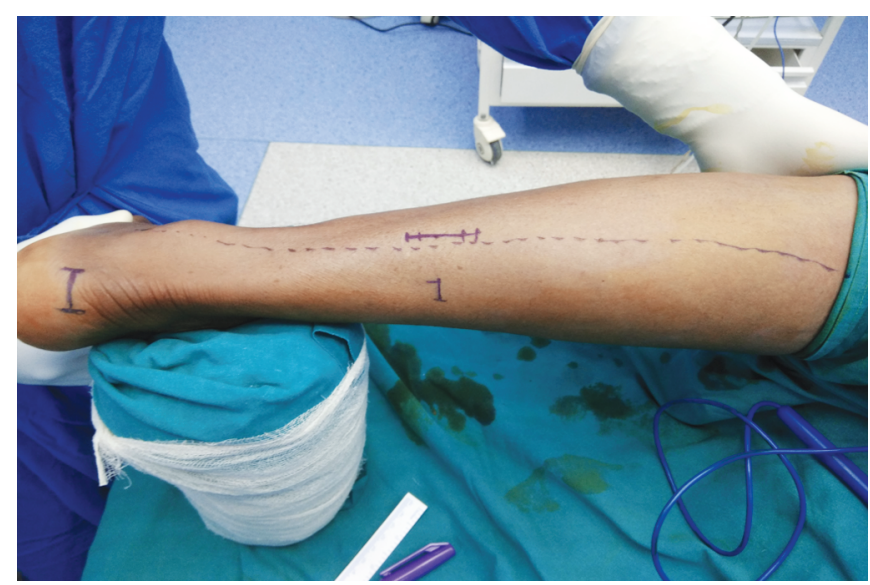

Fig. 1: Surface marking for sural nerve, insertion of tendo-Achilles, incision marking at the musculotendinous junction
American Orthopaedic Foot and Ankle Score was calculated at 6 weeks, 3 months, 6 months, and 9 months. Visual analog score was recorded at 9 months. At final follow-up at 9 months, patients were asked to choose satisfaction level in terms of high/partial/low and if they would recommend this surgery to their friends and would they go for the same for the opposite limb.

\section{Results}

The total number of patients was 20. The mean age of patients was 40.5 (range 28-48 years). Eight were females and 12 were males.

Preoperative VAS was $7.2 \pm 0.41$ (range 7-8). Postoperative VAS was $1.2 \pm 1.0$ (range $0-4)$. This difference comes out to be significant ( $p$ value $<0.001$ ) (Table 1).

American Orthopaedic Foot and Ankle Scores initially and at 6 weeks, 3 months, 6 months, and 9 months are shown in Table 2. A significant difference was present $(p$ value $<0.05$ ) at each follow-up compared to preoperative values and compared to the previous follow-up (Table 3).

In terms of patient satisfaction at 9 months of follow-up, 18 patients opted for high satisfaction and 2 opted for partial satisfaction. These 18 patients were ready to undergo surgery for the contralateral limb and would recommend it to family and friends (Table 4).

Mean preoperative dorsiflexion at the ankle was $2.9^{\circ}$ and mean post-op dorsiflexion was 16.35 at 3 months follow-up (Table 2). The difference comes out to be $13.45^{\circ}$.

\section{Discussion}

Chronic plantar fasciitis is a common condition and affects dayto-day life due to pain. Patients are often unsatisfied with the long duration of treatment and few of them are not relieved even after that. These are the cases in which gastrocnemius tightness should be looked for and the primary aim of the surgeon should be to give a pain-free heel.

Carlson et al. in their study on cadavers applied $500 \mathrm{~N}$ of force on tendo-Achilles and measured strain on the plantar fascia at

Table 1: VAS improved significantly postoperatively

\begin{tabular}{llll}
\hline & VAS $($ mean $\pm S D)$ & Median (range) & $p$ value \\
\hline Preoperative & $7.2 \pm 0.41$ & $7(7-8)$ & $<0.001$ \\
At 9 months & $1.2 \pm 1.0$ & $1(0-4)$ & \\
\hline
\end{tabular}

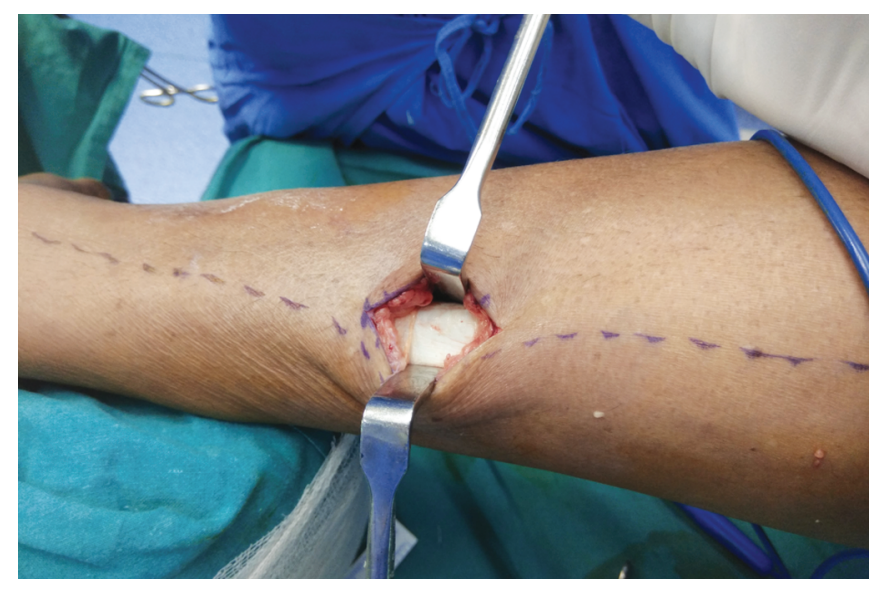

Fig. 2: Incision and sural nerve secured 
different angles of dorsiflexion at the metatarsophalangeal joint. They showed that dorsiflexion of the toes tightens the plantar fascia (the windlass effect) and increases the effect of tensile force of the tendo-Achilles on the plantar fascia. ${ }^{5}$ Chen et al. in their biomechanical model observed an increase in fascia strain by $66 \%$

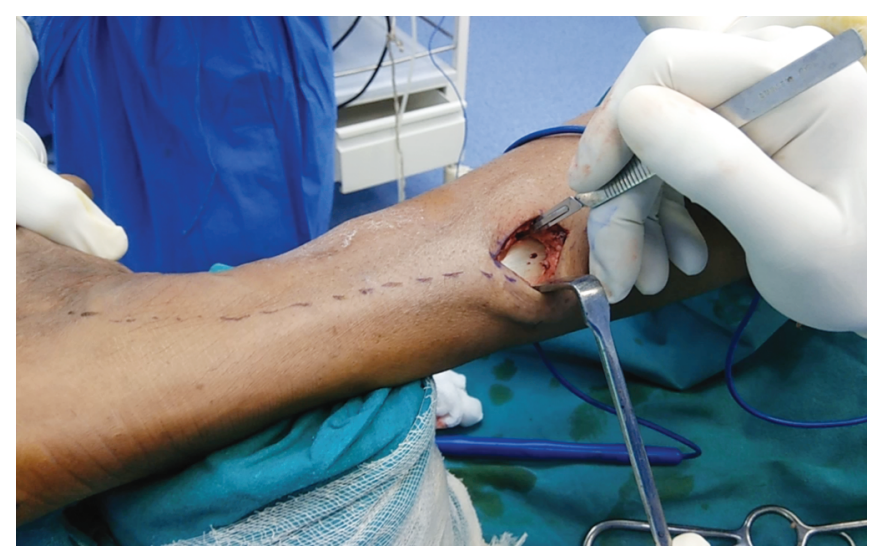

Fig. 3: Release of gastrocnemius at the musculotendinous junction

Table 2: Preoperative dorsiflexion and postoperative dorsiflexion at 3 months

\begin{tabular}{lll}
\hline S.no. & $\begin{array}{l}\text { Preoperative } \\
\text { dorsiflexion at ankle } \\
\text { (in degree) }\end{array}$ & $\begin{array}{l}\text { Postoperative dorsi- } \\
\text { flexion at 3 months } \\
\text { follow-up (in degree) }\end{array}$ \\
\hline 1 & 5 & 18 \\
2 & 4 & 20 \\
3 & 0 & 15 \\
4 & 5 & 21 \\
5 & 2 & 18 \\
6 & 5 & 22 \\
7 & 0 & 18 \\
8 & 0 & 14 \\
9 & 3 & 20 \\
10 & 5 & 20 \\
11 & 2 & 16 \\
12 & 6 & 24 \\
13 & 4 & 16 \\
14 & 0 & 15 \\
15 & 2 & 17 \\
16 & 3 & 18 \\
17 & 5 & 18 \\
18 & 5 & 21 \\
19 & 2 & 18 \\
20 & 0 & 16 \\
Mean & 2.9 & 16.35 \\
\hline & &
\end{tabular}

due to windlass mechanism and concluded that reducing heel rise and Achilles tendon force can reduce fascia loading which in turn can relieve pain due to plantar fasciitis. ${ }^{6}$ Patel and DiGiovanni found equinus contracture associated with $83 \%$ of acute plantar fasciitis and $82 \%$ of chronic cases. They also found isolated gastrocnemius contracture in $60 \%$ of acute and $52 \%$ of chronic cases. ${ }^{2}$ Cheung et al. found a linear increase in plantar fascia strain with increasing force on Achilles tendon and concluded that lengthening of Achilles tendon resulted in relief in plantar fascia strain. ${ }^{7}$ All these studies establish a firm relationship between Achilles tendon tension and plantar fascia strain and also show the role of Achilles lengthening for its treatment.

Lemont et al. in their study claimed plantar fasciitis is degenerative fasciosis without inflammation, not fasciitis and they questioned the role of NSAIDs and corticosteroid injections for its treatment. ${ }^{8}$ Though conservative treatment in the form of stretching exercises of the calf, plantar fascia specific stretching, ice massaging, night splints, and orthotics are helpful in most of the cases of isolated gastrocnemius tightness but not in all, and patients do feel relief in symptoms but not completely cured. ${ }^{9}$ American Orthopaedic Foot and Ankle Score has recommended the use of conservative treatment at least for 6 months before surgical intervention. We too managed our patients with conservative management initially. Radford et al. in their systemic review concluded that calf muscle stretching provides a small and statistically significant increase in ankle dorsiflexion but it is not clear whether the change is clinically important. ${ }^{10}$ We observed clinically significant improvement in VAS and AOFAS in all the patients. Plantar fascia release leads to lowering of arch and increase in the loading of other plantar ligaments and degeneration of the plantar fascia. Although earlier studies have claimed a good response with plantar fascia release, recent studies have shown overall poor outcomes with the procedure. ${ }^{11-13}$ Monteagudo et al. compared gastrocnemius recession to plantar fasciotomy for chronic plantar fasciitis and concluded that the gastrocnemius recession gives better outcomes and more patient satisfaction. ${ }^{14}$

Maskill et al. ${ }^{3}$ were the first ones to use gastrocnemius recession to treat plantar fasciitis. They obtained good results with the procedure. In their study, the VAS improved from 8.1 preoperatively to 1.9 postoperatively. They also reported that around $93 \%$ of patients were satisfied and would recommend this procedure to others. In our study, the VAS improved from 7.2 preoperatively to 1.2 postoperatively. Around $90 \%$ of patients who underwent the operation were highly satisfied and would recommend this procedure to others. Avila et al. reported a reduction of VAS from 8.1 to 0.8 and an increase in AOFAS from 47 to 91 . In their study, $90 \%$ of patients had high satisfaction. ${ }^{15}$ In a recently published study by Ficke et al., they have evaluated the outcome of gastrocnemius recession in obese patient's chronic plantar fascitis. In their study, VAS improved from 8 to 2 and $82 \%$ of patients wanted to

Table 3: AOFAS preoperative and at 6 weeks, 3 months, 6 months, 9 months follow-up

\begin{tabular}{|c|c|c|c|c|c|}
\hline \multirow[b]{2}{*}{ Time } & \multirow[b]{2}{*}{ AOFAS (mean $\pm S D)$} & \multirow[b]{2}{*}{ Median (range) } & \multicolumn{3}{|c|}{$p$ value } \\
\hline & & & Overall & Compared to preoperative & Compared to previous time \\
\hline Preoperative & $49.4 \pm 1.67$ & $50(47-51)$ & $<0.001^{*}$ & - & \\
\hline 6 weeks & $55.35 \pm 7.28$ & $52(50-75)$ & & $<0.05$ & $<0.05$ \\
\hline 3 months & $74.35 \pm 4.69$ & $75(56-79)$ & & $<0.05$ & $<0.05$ \\
\hline 6 months & $84.55 \pm 3.43$ & $86(75-89)$ & & $<0.05$ & $<0.05$ \\
\hline 9 months & $93.3 \pm 6.17$ & 95 (75-99) & & $<0.05$ & $<0.05$ \\
\hline
\end{tabular}


Table 4: Outcome in terms of patient satisfaction at 9 months follow-up

\begin{tabular}{ll}
\hline Patient satisfaction & No. of patients \\
\hline High & 18 \\
Partial & 2 \\
Low & 0 \\
\hline
\end{tabular}

recommend this procedure to others. ${ }^{4}$ Hoefnagels et al. studied 75 cases with chronic plantar fasciitis. Out of these, 32 patients were operated on with gastrocnemius recession. Postoperatively, they found an increase of dorsiflexion at the ankle joint by $16^{\circ}$. They too reported excellent patient satisfaction rates. ${ }^{16}$ In our study, we have found an increase in dorsiflexion by $13.45^{\circ}$. In a similar study conducted by Molund et al., VAS improved from 7 to 1.8 , and 14 patients out of 18 were satisfied with the surgery and would recommend it to others. ${ }^{17}$ Similar results were reported by Abbassian et al. ${ }^{18}$ All these studies show the consistency of results that surgeons have got with this procedure.

We performed gastrocnemius recession at the musculotendinous junction. Approach at this level directly puts the sural nerve at risk. Maskill et al. ${ }^{3}$ in their study performed gastrocnemius recession at musculotendinous junction and reported good results. They did not report any case of sural nerve injury in their study. Another approach for gastrocnemius recession is at the proximal level where the medial head of the gastrocnemius is released. This approach puts great saphenous vein and saphenous nerve at risk. Monteagudo et al. ${ }^{14}$ used this approach and reported a good outcome. Molund et al. reported a higher complication rate with this approach. ${ }^{17}$

In all cases, the sural nerve was identified and secured in our study. We did not face any complications related to the sural nerve. There were no complaints related to wound or scar. None of the patients had postoperative calf muscle weakness.

Future studies need to compare gain in ankle dorsiflexion between both conservative and operative groups. Randomized controlled trials of this procedure can provide a better level of evidence of its effectiveness.

\section{CONCLUSION}

Gastrocnemius recession done for isolated gastrocnemius tightness causing chronic plantar fasciitis provides statistically significant improvement in symptoms without any major complications. It should definitely be considered in patients not responding to a conservative approach.

\section{References}

1. Riddle DL, Pulisic M, Pidcoe $P$, et al. Risk factors for plantar fasciitis: a matched case-control study. J Bone Joint Surg Am 2003;85-A(5):872877. DOI: 10.2106/00004623-200305000-00015.
2. Patel A, DiGiovanni B. Association between plantar fasciitis and isolated contracture of the gastrocnemius. Foot Ankle Int 2011;32(1):5-8. DOI: 10.3113/FAI.2011.0005.

3. Maskill JD, Bohay DR, Anderson JG. Gastrocnemius recession to treat isolated foot pain. Foot Ankle Int 2010;31(1):19-23. DOI: 10.3113/ FAI.2010.0019.

4. Ficke B, Elattar O, Naranje SM, et al. Gastrocnemius recession for recalcitrant plantar fasciitis in overweight and obese patients. Foot Ankle Surg 2018;24(6):471-473. DOI: 10.1016/j.fas.2017.05.008.

5. Carlson RE, Fleming LL, Hutton WC. The biomechanical relationship between the tendoachilles, plantar fascia and metatarsophalangeal joint dorsiflexion angle. Foot Ankle Int 2000;21(1):18-25. DOI: $10.1177 / 107110070002100104$

6. Chen YN, Chang CW, Li CT, et al. Finite element analysis of plantar fascia during walking: a quasi-static simulation. Foot Ankle Int 2015;36(1):90-97. DOI: 10.1177/1071100714549189.

7. Cheung JTM, Zhang M, An KN. Effect of Achilles tendon loading on plantar fascia tension in the standing foot. Clin Biomech 2006;21(2):194-203. DOI: 10.1016/j.clinbiomech.2005.09.016.

8. Lemont $\mathrm{H}$, Ammirati KM, Usen N. Plantar fasciitis. A degenerative process (fasciosis) without inflammation. J Am Podiatr Med Assoc 2003;93(3):234-237. DOI: 10.7547/87507315-93-3-234.

9. League AC. Current concepts review: plantar fasciitis. Foot Ankle Int 2008;29(3):358-366. DOI: 10.3113/FAI.2008.0358.

10. Radford JA, Burns J, Buchbinder R, et al. Does stretching increase ankle dorsiflexion range of motion? A systematic review. Br J Sports Med 2006;40(10):870-875. DOI: 10.1136/bjsm.2006.029348discussion 875.

11. Gefen A. Stress analysis of the standing foot following surgical plantar fascia release. J Biomech 2002;35(5):629-637. DOI: 10.1016/ s0021-9290(01)00242-1.

12. Kim W, Voloshin AS. Role of plantar fascia in the load bearing capacity of the human foot. J Biomech 1995;28(9):1025-1033. DOI: 10.1016/0021-9290(94)00163-x.

13. Cheung JTM, An KN, Zhang M. Consequences of partial and total plantar fascia release: a finite element study. Foot Ankle Int 2006;27(2):125-132. DOI: 10.1177/107110070602700210.

14. Monteagudo M, Maceira E, Garcia-Virto V, et al. Chronic plantar fasciitis: plantar fasciotomy versus gastrocnemius recession. Int Orthoped 2013;37(9):1845-1850. DOI: 10.1007/s00264-013-2022-2.

15. Avila A, Monteagudo M, Martinez-Albornoz P, et al. Medial gastrocnemius proximal release in chronic plantar fasciitis. Prospective study of 75 cases. Foot Ankle Surg 2016;2(22):24-25. DOI: 10.1016/j.fas.2016.05.049.

16. Hoefnagels E, Weereheijm L, Louwerens JW, et al. Chronic therapy resistant plantar fasciitis, the effect of lengthening the gastrocnemius muscle. Foot Ankle Surg 2016;22(2):58. DOI: 10.1016/j.fas.2016.05.143.

17. Molund $M$, Paulsrud $\varnothing$, Ellingsen Husebye $E$, et al. Results after gastrocnemius recession in 73 patients. Foot Ankle Surg 2014;20(4):272-275. DOI: 10.1016/j.fas.2014.07.004.

18. Abbassian A, Kohls-Gatzoulis J, Solan MC. Proximal medial gastrocnemius release in the treatment of recalcitrant plantar fasciitis. Foot Ankle Int 2012;33(1):14-19. DOI: 10.3113/FAI.2012.0014. 